\title{
BMJ Open Efficacy, safety and response predictors of adjuvant astragalus for diabetic kidney disease (READY): study protocol of an add-on, assessor-blind, parallel, pragmatic randomised controlled trial
}

\author{
Kam Wa Chan (D) , ${ }^{1}$ Alfred Siu Kei Kwong, ${ }^{2}$ Pun Nang Tsui, ${ }^{3}$ \\ Simon Chi Yuen Cheung, ${ }^{4}$ Gary Chi Wang Chan, ${ }^{1}$ Wing Fai Choi, ${ }^{5}$ Wai Han Yiu, \\ Yanbo Zhang, ${ }^{5}$ Michelle Man-Ying Wong, ${ }^{3}$ Zhang-Jin Zhang, ${ }^{5}$ \\ Kathryn Choon Beng Tan, ${ }^{1}$ Lixing Lao, ${ }^{5,6}$ Sydney Chi Wai Tang (i]) ${ }^{1}$
}

To cite: Chan KW, Kwong ASK, Tsui PN, et al. Efficacy, safety and response predictors of adjuvant astragalus for diabetic kidney disease (READY): study protocol of an add-on, assessor-blind, parallel, pragmatic randomised controlled trial. BMJ Open 2021;11:e042686. doi:10.1136/ bmjopen-2020-042686

- Prepublication history and additional materials for this paper is available online. To view these files, please visit the journal online (http://dx.doi. org/10.1136/bmjopen-2020042686).

Received 11 July 2020 Revised 07 December 2020 Accepted 11 December 2020

Check for updates

(c) Author(s) (or their employer(s)) 2021. Re-use permitted under CC BY-NC. No commercial re-use. See rights and permissions. Published by BMJ.

For numbered affiliations see end of article.

Correspondence to

Professor Sydney Chi Wai Tang; scwtang@hku.hk

\section{ABSTRACT}

Introduction Diabetic kidney disease (DKD) is a prevalent and costly complication of diabetes with limited therapeutic options, being the leading cause of end-stage kidney disease in most developed regions. Recent big data studies showed that add-on Chinese medicine (CM) led to a reduced risk of end-stage kidney disease and mortality among patients with chronic kidney disease (CKD) and diabetes. Astragalus, commonly known as huang-qi, is the most prescribed CM or used dietary herb in China for diabetes and DKD. In vivo and in vitro studies showed that astragalus ameliorated podocyte apoptosis, foot process effacement, mesangial expansion, glomerulosclerosis and interstitial fibrosis. Nevertheless, the clinical effect of astragalus remains uncharacterised. This pragmatic clinical trial aims to evaluate the effectiveness of add-on astragalus in patients with type 2 diabetes, stage 2-3 CKD and macroalbuminuria, and to identify related response predictors.

Methods and analysis This is an add-on, assessorblind, parallel, pragmatic randomised controlled clinical trial. 118 patients diagnosed with DKD will be recruited and randomised 1:1 to receive 48 weeks of add-on astragalus or standard medical care. Primary endpoints are the changes in estimated glomerular filtration rate and urine albumin-to-creatinine ratio between baseline and treatment endpoint. Secondary endpoints include adverse events, fasting blood glucose, glycated haemoglobin, lipids and other biomarkers. Adverse events are monitored through self-complete questionnaire and clinical visits. Outcomes will be analysed by regression models. Subgroup and sensitivity analyses will be conducted for different epidemiological subgroups and statistical analyses. Enrolment started in July 2018.

Ethics and dissemination This study was approved by the Institutional Review Board of the University of Hong Kong/Hospital Authority Hong Kong West/East/Kowloon Central clusters (UW 16-553/HKEC-2019-026/REC (KC/ KE)-19-0049/ER-4). We will report the findings in medical journals and conferences. The dataset will be available on reasonable request.

\section{Strengths and limitations of this study}

- Existing epidemiological data suggested that Chinese medicine (CM) was associated with retarded progression of renal function among diabetic kidney disease (DKD) patients. It is timely to perform a clinical trial on astragalus, the most used herbs in diabetes and DKD with unclear clinical effectiveness.

- The inclusion/exclusion criteria, primary outcome measurement and the corresponding analyses are designed according to conventionally used parameters to facilitate further meta-analysis with other clinical studies for a wide range of audience.

- A responder analysis is built into the trial as the secondary analysis to identify possible factors (including biomarkers and symptom-based diagnosis) that could lead to more personalised the use of astragalus.

- We conducted a focus group interview series to explore the expectations of patients and clinicians (both conventional and $\mathrm{CM}$ ) to refine the study design (drug form, dosage, administration route, frequency, health services delivery and outcome measurement) for better clinical translation.

- As the trial is open-label, subjective outcomes including quality of life could not be assessed.

Trial registration number NCT03535935

\section{INTRODUCTION}

In 2019, it was estimated that 463 million $(9.3 \%)$ people were living with diabetes worldwide and the figure was projected to reach 578 million by 2030 , with the highest prevalence in North America at present. ${ }^{1}$ In 2017, the healthcare expenditure on diabetes reached US $\$ 850$ billion globally $(11.6 \%$ of global health expenditure). ${ }^{2}{ }^{3}$ Diabetic 
kidney disease (DKD) refers to the chronic kidney disease (CKD) caused by long-standing diabetes. DKD presents in more than one third of all diabetic patients and is the leading cause of end-stage kidney disease in many developed regions which requires replacement therapy in the form of dialysis and transplantation. ${ }^{45}$ In Hong Kong, the incidence of diabetes-related end-stage kidney disease increased from $26.2 \%$ in 1996 to $49.6 \%$ in $2013^{6}$ and endstage kidney disease increased 5.23 times the annual direct medical cost to the local public health system. ${ }^{7}$ Furthermore, DKD was accounted for $23.4 \%$ (31.1\% vs $7.7 \%$ ) absolute increase in 10-year mortality in the $\mathrm{USA}^{8}$ and 16 years shorter life expectancy in Taiwan ${ }^{9}$ when compared with those without diabetes and kidney diseases.

The risk factors and pathogenesis of DKD are heterogeneous ${ }^{5}$ involving metabolic, ${ }^{1011}$ inflammatory ${ }^{12-14}$ haemodynamic ${ }^{15-18}$ and many other pathways. ${ }^{519}$ Thickening of glomerular basement membrane, mesangial expansion, effacement of foot process, formation of KimmelstielWilson nodules, glomerulosclerosis and interstitial fibrosis are the classical histopathological features of diabetic nephropatchy.$^{15}{ }^{20}$ Conventional blockade on the renin-angiotensin-aldosterone system (RAAS) offers limited effect on clinical outcomes. ${ }^{21-24}$ In a previous meta-analysis of 9797 patients with stage 3-5 CKD, RAAS blockade did not reduce all-cause mortality and only provided a mild risk reduction in the composite endpoint of replacement therapy initiation or doubling of serum creatinine when compared with placebo or other antihypertensive agents. ${ }^{22}$ RAAS blockade with combined ACE inhibitor (ACEI) and angiotensin II receptor blocker (ARB) resulted in increased adverse events but not the expected synergistic effect. ${ }^{2325}$ More therapies with different working mechanisms are needed.

Chinese medicine (CM) has been extensively used among patients with diabetes and DKD in Asia. ${ }^{2627}$ Previous observational studies from Taiwan with 47876 and 24971 subjects showed that the use of add-on prescribed CM is associated with $40 \%$ reduction of mortality ${ }^{27}$ and $59 \%$ risk reduction of end-stage kidney disease, respectively. ${ }^{26}$ Astragalus membranaceus, commonly known as huang-qi, is the most frequently used CM or dietary herb for DKD. ${ }^{28}$ Systematic reviews showed that astragalus could enhance creatinine clearance, reduce albuminuria and reduce blood pressure among patients with CKD and DKD. ${ }^{29-31}$ Meta-analysis also showed that astragalus' effect in improving renal clearance and reducing albuminuria was better than routine care (without ACEI or ARB) and the efficacy was comparable to ACEI or ARB. ${ }^{31}$ In vivo and in vitro evidence suggested that astragaloside IV, an active ingredient of astragalus, could ameliorate podocyte apoptosis, foot process effacement, mesangial expansion, glomerulosclerosis and interstitial fibrosis through regulating the nuclear factor kappa B (NF-kB) and transforming growth factor beta 1 (TGF- $\beta_{1}$ ) signalling pathways, which partly explained the renoprotective effect. $^{32}{ }^{33}$ Nevertheless, the methodological reporting and quality of the existing clinical trials were inadequate and further evaluation is needed. Based on our preliminary result of ongoing trials, CM formulations containing astragalus are likely to retard the progression of DKD. ${ }^{3435}$ Considering the extensive current use of astragalus, clinical study could be considered before preclinical investigation as suggested by the WHO. ${ }^{36}$

\section{METHODS/DESIGN \\ Objective}

This pragmatic clinical trial aims to evaluate the effectiveness of add-on astragalus on patients with type 2 diabetes with stage 2-3 chronic kidney disease and macroalbuminuria and to identify related response predictors for subsequent large-scale health services research.

\section{Study design}

Add-on, assessor-blind, parallel, pragmatic randomised controlled trial. The WHO Trial Registration Data Set (online supplemental appendix 1) and Standard Protocol Items: Recommendations for Interventional Trials (SPIRIT) checklist (online supplemental SPIRIT checklist) are enclosed.

\section{Inclusion and exclusion criteria}

Patients with (1) type 2 diabetes for at least 5 years; (2) estimated glomerular filtration rate (GFR) $\geq 30$ and < $90 \mathrm{~mL} / \mathrm{min} / 1.73 \mathrm{~m}^{2}$ confirmed by repeated testing over 3 months calculated by the abbreviated Modification of Diet in Renal Disease (MDRD) study equation ${ }^{37} 38$; (3) persistent macroalbuminuria with spot urine albumin-tocreatinine ratio (UACR) $\geq 300 \mathrm{mg} / \mathrm{g}$ confirmed by at least two consecutive first morning void urine samples; (4) age between 35 and 80 years old; (5) stable dose of antidiabetic agent(s) including insulin for at least 12 weeks and (6) stable dose of ACEI or ARB for at least 12 weeks will be recruited.

Patients will be excluded if with (1) UACR $\geq 5000 \mathrm{mg} / \mathrm{g}$; (2) a known history of glomerulonephritis, polycystic kidney disease, systemic lupus erythematosus or any suggestive evidence of non-diabetic glomerulopathy; (3) known history of kidney transplant; (4) concurrent severe disorders of heart, brain, liver and hematopoietic system, tumour, mental disorder; (5) deranged liver function; (6) poorly controlled blood pressure; (7) known history of intolerance or malabsorption of oral medications; (8) uncontrollable urinary infection; (9) experiencing pregnancy and (10) participating in other clinical trial(s) within 30 days.

\section{Sample size calculation}

Since the primary objective of this trial is to evaluate key clinical outcomes and to perform a preliminary analysis on potential response predictors, we calculated the sample size based on the control of inflation factor (IF) to the estimation of sample size for the subsequent largescale studies. ${ }^{39} 40$ One hundred and eighteen patients (around 60 per group) are needed. 


$$
\begin{gathered}
\mathrm{IF}=\mathrm{S}_{\mathrm{ucl}} / \mathrm{S}_{\mathrm{obs}}=\operatorname{sqrt}\left[(\mathrm{n}-1) / \mathrm{x}_{1-\alpha, \mathrm{n}-1}^{2}\right] \\
\mathrm{N}_{\mathrm{adj}} / \mathrm{N}_{\mathrm{unadj}} \approx \mathrm{IF}^{2} \approx \mathrm{n}_{\text {unadj }} \times \mathrm{IF}^{2} \\
\left.\mathrm{~N} \approx\left[2\left(Z_{1-\alpha / 2}+Z_{1-\beta}\right)^{2}(I F * s)^{2}\right] /\left(\mu_{1}-\mu_{2}\right)^{2}\right] \\
\left.=\left[2\left(Z_{1-\alpha / 2}+Z_{1-\beta}\right) s^{2}\right] /\left(\mu_{1}-\mu_{2}\right)^{2}\right] \\
\mathrm{Z}_{1-\beta}=\mathrm{Z}_{1-\alpha / 2}\left(\mathrm{IF}^{-1}-1\right)+\mathrm{Z}_{1-\beta} * \mathrm{IF}^{-1}
\end{gathered}
$$

where

IF=Inflation factor.

$\mathrm{S}_{\mathrm{ucl}}=\mathrm{SD}$ of upper CI.

$\mathrm{S}_{\mathrm{obs}}=$ Observed SD in pilot study.

$\alpha=$ Chosen confidence level.

$\beta=$ Nominal power set for main study.

$\beta^{\prime}=$ Actual power achieved for main study by using pilot

SD for sample size calculation.

$\mathrm{n}=$ Sample size of pilot study.

$\mathrm{N}=$ Sample size of main study.

$\mathrm{N}_{\text {unadj }}=$ Sample size of main study with no adjustment on SD.

$\mathrm{N}_{\mathrm{adi}}=$ Sample size of main study with adjustment on SD.

The SD used for sample size calculation for large-scale main studies is often underestimated by small-scale pilot studies; therefore, an IF is needed for adjustment in sample size calculation. ${ }^{39}{ }^{40}$ IF is calculated based on the size of pilot study and the confidence level of achieving at least the desired power in subsequent main studies. Therefore, the actual achieved power of the main studies depends on the nominal power set for the main study and the IF.

In order to be $95 \%$ confident (two-sided) that the main study achieves a power of $70 \%$ with nominal power set at $80 \%$ (ie, a $10 \%$ power forfeit), the IF should be controlled to less than 1.13. At IF $=1.13$, a sample size of 100 is therefore needed to attain $95 \%$ one-sided confidence that the main studies will achieve the nominal power to test the hypothesis of add-on astragalus could be more effective in stabilising the GFR among patients with DKD when compared with standard care. To allow a $15 \%$ attrition rate, a sample size of 118 patients is therefore needed for this pilot study.

Currently, there is limited evidence on the symptombased response predictors of astragalus. A general recommendation for power estimation is to have 10 events per variable. ${ }^{41}$ From the previous systematic review, we estimate that around $60 \%$ of patients will have stabilised GFR after receiving astragalus. ${ }^{31}$ One hundred and eighteen subjects with $15 \%$ attrition will power up to six variables for the screening of predictors. A univariable screening on the 11 prespecified potential symptom-based predictors will be conducted to reduce the number of predictors for the subsequent multivariable regression analysis, in order to maximise the power of the regression analysis.

\section{Recruitment and randomisation}

Patients will be recruited from general and specialist outpatient clinics of Queen Mary Hospital, Queen Elizabeth Hospital, Hospital Authority Hong Kong East Cluster through consultations and the community via public health campaigns. The details of study will be explained by principal investigators (PIs) or co-investigators (CoIs) before written consent is obtained from each participating patient. All patients will undergo a 2-week run-in period, during which the dosage of their medications will be stabilised. Blood and urine samples will be sent to an independent local laboratory for screening. Patients are considered eligible for the study if their liver functions are normal and fulfil the inclusion criteria. Recruitment started in July 2018 and the recruitment is ongoing.

A random sequence was generated and encrypted with computer by an independent staff of the University of Hong Kong and kept in sealed opaque envelopes. The password of the sequence is kept in a sealed, duly signed opaque envelop locked by research assistants (RAs). The allocation sequence is concealed from PIs, Co-Is, CM physicians and all research staffs that are responsible for patient screening, randomisation or sample analysis. Eligible patients will be randomised 1:1 to either receive active intervention along with standard care or standard care alone. The allocation is masked from the outcome assessor (technicians from an independent laboratory). The study subjects could not be masked due to the nature of treatment. Since the primary clinical outcomes under investigation are objectively assessed and the outcome assessor is blinded, placebo effect and outcome measurement bias should be minimised. The flow of study is presented in figure 1. Under no circumstances the primary outcome assessors will be unblinded.

\section{Intervention and control}

The intervention under investigation is astragalus. Patients under intervention will receive astragalus daily on top of standard medical care for 48 weeks. The CM physicians will advise on the dose and possible adverse

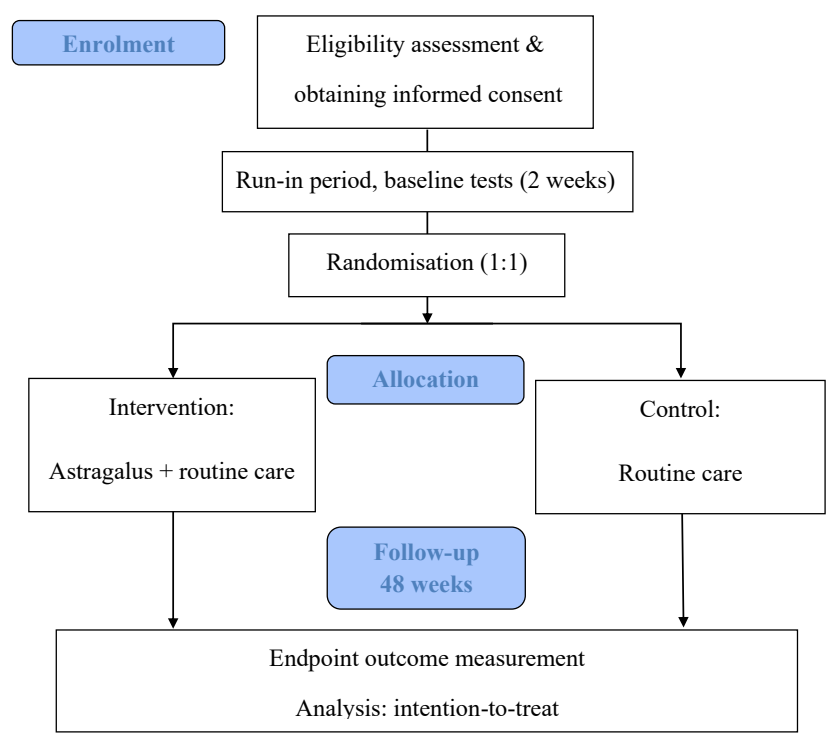

Figure 1 The flow of research. 
events of astragalus based on his/her professional knowledge. Existing literature supports a safe dosage of raw astragalus from $15 \mathrm{~g} /$ day to $50 \mathrm{~g} /$ day. ${ }^{42}{ }^{43}$ According to the China Pharmacopeia, the recommended therapeutic dosage of astragalus is below $30 \mathrm{~g} /$ day. To ensure the safety of patients, CM physicians are reminded not to propose dosage exceeding $30 \mathrm{~g} /$ day. All patients will continue their standard medication and follow-up with the same consultation schedule with CM physicians. Standard care is used as control to best reflect the real-world practice and the future application scenario of this trial. ${ }^{44}$

\section{Herbal safety}

Soluble herbal granules prepared by PuraPharm (listed in US Pharmacopeia as dietary ingredient: VER-DI-PUR-09) are used. The production process is in strict compliance with standards of Good Manufacturing Practice. Fully registered CM physicians from the School of Chinese Medicine, The University of Hong Kong, will be responsible for the clinical diagnosis and prescription. After 4-6 weeks of randomisation, all patients will undergo liver function tests and renal function tests to monitor acute changes of renal and liver function.

\section{Outcome measurement}

The primary outcome measures are the changes of estimated $\mathrm{GFR}^{45}$ and UACR from baseline (week 0) to treatment endpoint (week 48). As the progression of kidney disease is slow, we believe reporting 1-year (48-week) change in GFR is necessary to avoid extrapolation while extended observation may lead to substantial attrition and is limited by resources. Secondary outcome measures include adverse events and changes in CKD stage, haemoglobin $\mathrm{A}_{1 \mathrm{c}}\left(\mathrm{HbA}_{1 \mathrm{c}}\right)$, lipids, urinary monocyte chemotactic protein 1 (MCP-1) and urinary cystatin $\mathrm{C}$ from baseline to the midpoint (week 24) and the end of treatment.

\section{Data collection}

Patient demographics including age, gender, body mass index (BMI), duration of diabetes, other medical history and concurrent medications will be retrieved by the electronic clinical management system of hospital authority by Co-Is and RAs. Estimated GFR, UACR, HbA, lipids and liver function tests will be assessed by an independent laboratory (Chan \& Hou Medical Laboratories Limited) which is accredited by College of American Pathologists, Royal College of Pathologists Australasia and Quality Control for Molecular Diagnostics, UK. MCP-1 and cystatin $\mathrm{C}$ will be assessed at lead PI's research laboratory by an independent RA with commercially available kits. Blood pressure will be taken during consultation. Blood and urine samples will be taken at an overnight ( $>8$ hours) fasting state.

Estimated GFR will be calculated using the MDRD equation with serum creatinine, age, ethnicity and gender. Clinical presentations and CM symptom-based diagnosis will be assessed in a structured consultation developed for this purpose. To ensure consistency and reliability of assessment and to minimise bias from investigators across the study, only three synchronised CM physicians will assess the patients.

A self-complete questionnaire will be distributed to the subjects to monitor adverse events, and they are advised to inform the PIs, Co-Is, CM physicians or RAs immediately if adverse events arise. All adverse events will be coded based on Common Terminology Criteria for Adverse Events (CTCAE) 5.0, following the recommendation of Consolidated Standards of Reporting Trials extension for Chinese herbal medicine formulas. ${ }^{46}$

Follow-up consultations will be held for all patients bi-weekly in the first month and monthly subsequently until the end of treatment for all patients. Minor adjustments are allowed based on clinical needs. Evaluation of outcomes will be performed at baseline, week 24 (treatment midpoint) and week 48 (end of treatment). The follow-up schedule is summarised in table 1.

\section{Data management}

A trial management committee (TMC) formed by lead PI, Co-Is and RAs will centralise all trial data. Co-Is and RAs will collect, clean and send the data to TMC weekly. All data will be double entered, secured and cleaned before analysis to prevent data entry errors. TMC will have regular meetings monthly to discuss the progress and double check the data of the trial. Only PI, Co-Is and regulatory bodies will have access to the patient data to protect data privacy. An independent Data and Safety Monitoring Board (DSMB) (VCH Chung, W Wong, JWF Yeung) has been established with expert in methodology, biostatistics and clinical medicine to monitor the progress of the trial, including adverse events and change in protocol. DSMB will have meetings one to two times a year. No competing interests have been reported from DSMB. Trial result will be published in academic journal and trial subjects will be notified.

\section{Handling of withdraw and dropout}

In order to maximise subjects' compliance, we will provide a triple thorough consent process for all participants covering details of the study schedule, potential side effects of treatment and the responsibilities of the subjects. An independent e-mail account and a direct telephone line is available for this study to enable active communication with patients. Extra visits will be arranged for patients if necessary. To monitor the adherence of study medication, we will arrange irregular visits for patients and count the unfinished medication.

\section{Termination criteria}

The treatment will be terminated for a specific subject if he/she (1) develops serious adverse event (SAE); (2) develops hypersensitivity towards astragalus and (3) participates in other clinical trial. The whole study will be terminated under the following circumstances: (1) presence of clustered $\mathrm{SAE}(\mathrm{s})$ related to astragalus with 
Table 1 Follow-up schedule

\begin{tabular}{|c|c|c|c|c|c|}
\hline \multirow[b]{3}{*}{ Timepoint } & \multicolumn{5}{|c|}{ Study period } \\
\hline & \multirow{2}{*}{$\begin{array}{l}\text { Enrolment } \\
\text { Before } \\
\text { treatment }\end{array}$} & \multirow{2}{*}{$\begin{array}{l}\text { Allocation } \\
\text { Week 0, Day } 1\end{array}$} & \multicolumn{3}{|c|}{ Post allocation-treatment period } \\
\hline & & & $\begin{array}{l}\text { Week 1-4 ( } \pm 3 \\
\text { days) }\end{array}$ & $\begin{array}{l}\text { After } 4-6 \text { weeks } \\
\text { ( } \pm 7 \text { days) }\end{array}$ & $\begin{array}{l}\text { After } 24 \text { and } 48 \\
\text { weeks ( } \pm 7 \text { days) }\end{array}$ \\
\hline \multicolumn{6}{|l|}{ Enrolment } \\
\hline Eligibility screen & $\mathrm{X}$ & & & & \\
\hline Allocation & & $x$ & & & \\
\hline \multicolumn{6}{|l|}{ Interventions } \\
\hline Intervention (interventional group) & & $x$ & $x$ & $x$ & $x$ \\
\hline Routine care (all patients) & & $x$ & $\mathrm{X}$ & $x$ & $\mathrm{X}$ \\
\hline $\begin{array}{l}\text { Blood pressure, weight, hip-waist } \\
\text { circumference }\end{array}$ & & $\mathrm{X}$ & $x$ & $x$ & $x$ \\
\hline Demographics & & $x$ & & & \\
\hline Clinical presentations & & $x$ & $x$ & $x$ & $x$ \\
\hline Adverse events & & $X$ & $\mathrm{X}$ & $X$ & $X$ \\
\hline
\end{tabular}

supportive evidence and (2) completion of all follow-up assessments.

SAE includes adverse events that result in death, require either hospitalisation or the prolongation of hospitalisation, are life-threatening, result in a persistent or significant disability/incapacity, result in a congenital anomaly/ birth defect or events classified as grade 3 or above in CTCAE 5.0. Other important medical events, based on appropriate medical judgement, may also be considered SAEs if a patient's health is at risk and intervention is required to prevent an outcome mentioned.

\section{Data analysis}

Missing values will be imputed by multiple regression. The analysis will follow intention-to-treat principle that all randomised patients will be included in the analysis. STATA and GraphPad Prism will be used for the analysis.

Demographics will be presented as mean $\pm \mathrm{SD}$ or percentage. UACR will be log-transformed and reported as geometric means. Smoking history will be stratified into non-smoker, ex-smoker and current smoker. Rapid renal progression is predefined as a consecutive annual GFR drop of over $5 \mathrm{~mL} / \mathrm{min} / 1.73 \mathrm{~m}^{2}$ or a cumulative GFR drop of over $25 \mathrm{~mL} / \mathrm{min} / 1.73 \mathrm{~m}^{2}$ for 5 years. ${ }^{47} 48$ Differences in mean and proportion between groups will be tested by t-test and $\chi^{2}$ test.

Mixed regression models will be used to compare the rate of change in estimated GFR and UACR. Analysis of covariance will be used to compare the adjusted mean of outcomes at week 48 between intervention group and control group with the corresponding baseline values as covariates. Data will be presented as the difference in adjusted means between the groups with $95 \%$ CI and the corresponding $\mathrm{p}$ value.

The adverse events will be recorded according to CTCAE 5.0 and categorised into five grades (grade 1: mild, asymptomatic or mild symptoms, clinical or diagnostic observations only, no intervention indicated; grade 2: moderate, minimal, local or non-invasive intervention indicated, limiting age-appropriate instrumental activities of daily living; grade 3: severe or medically significant but not immediately life-threatening, hospitalisation or prolongation of hospitalisation indicated, disabling, limiting self-care activities of daily living; grade 4: lifethreatening consequences, urgent intervention indicated and grade 5: death related to adverse events). The percentage of all adverse events with more than one case will be compared between groups. SAE will be analysed case by case descriptively.

To minimise type I error inflation, the analysis will follow a hierarchical approach in the order of (1) comparison of baseline to end of treatment on estimated GFR and UACR; (2) comparison of baseline to end of treatment on other outcome measurements; (3) comparison of baseline to treatment midpoints on estimated GFR and UACR and (4) comparison of baseline to treatment midpoints on other outcome measurements.

For the assessment of predictive factors as secondary analysis, the dependent variable will be the treatment response which is categorised into:

1. Improved or stabilised renal function, defined as estimated GFR after 48-week treatment being higher or equal to baseline. 
2. Non-responder, defined as patients having estimated GFR decreased at a rate of less than $5 \mathrm{~mL} / \mathrm{min} / 1.73 \mathrm{~m}^{2}$ after 48-week treatment compared with baseline.

3. Rapid deteriorating renal function, defined as estimated GFR of more than $8 \mathrm{~mL} / \mathrm{min} / 1.73 \mathrm{~m}^{2}$ after 48-week treatment compared with baseline.

Potential prognostic variables (baseline values) will include:

1. Demographics: age, gender, BMI, systolic blood pressure, history and duration of smoking and alcohol consumption.

2. Symptom-based diagnosis: presence of CM-based symptom-based subtype (eg, spleen and kidney qi deficien$c y)$ based on the presentation of standardised and commonly documented signs and symptoms. ${ }^{49}$

3. Biochemical profile: GFR, UACR, $\mathrm{HbA}_{1 \mathrm{c}}$, lipids.

All potential predictors will first be included into univariable regression models followed by multivariable stepwise regression analysis. Variables that are not significant at a $5 \%$ level will be excluded.

Subgroup analyses will be performed for (1) CKD stages stratified into stages $2,3 \mathrm{a}$ and $3 \mathrm{~b}^{50}$; (2) UACR levels stratified by $100 \mathrm{mg} / \mathrm{mmol}^{51}$; (3) gender and (4) age groups. Sensitivity analyses will be performed for (1) per-protocol cohort; (2) estimation of GFR by c-MDRD ${ }^{52}$ and Chronic Kidney Disease Epidemiology Collaboration (CKD-EPI) ${ }^{53}$ equations; (3) missing data imputed with last-observationcarried-forward and (4) different analytical approaches (change score) and categorisations of primary outcomes.

\section{Patient and public involvement}

We conducted a focus group interview series to collate the experience and expectations of patients and clinicians (both conventional medicine and $\mathrm{CM}$ ) on the study design (drug form, dosage, administration route, frequency, health services delivery and outcome measurement) for this trial. ${ }^{54}$ The study results will be disseminated to diabetes patient groups and the participants via public workshops and talks.

\section{ETHICS AND DISSEMINATION}

This study was approved and monitored by the Institutional Review Board of the University of Hong Kong/ Hospital Authority Hong Kong West/East/Kowloon Central clusters (Ref: UW 16-553/HKEC-2019-026/REC (KC/KE)-19-0049/ER-4). The patient information sheet and consent form are enclosed in Appendix 2. Result will be disseminated as conference presentations and journal publications on completion.

\section{DISCUSSION}

Diabetes and DKD are significant public health burdens, and astragalus is the most used herbs among these patients with unclear clinical effectiveness. There is an urgent need to characterise the effect and the associated response predictors of astragalus to prevent unnecessary consumption and increase the cost-effectiveness of administration. Also, the assessment of response predictors of both biomarkers and symptom-based factors will facilitate the integration and clinical translation of generated evidence between conventional medicine and CM physicians. Based on our preliminary result of ongoing trials, CM formulations containing astragalus are likely to retard the progression of DKD. ${ }^{345}$ This trial aims to evaluate the effect of astragalus and identify related response predictors for more personalised application and further large-scale health services research.

To facilitate further meta-analysis with other clinical studies for wide range of audience, the inclusion/ exclusion criteria, primary outcome measurement and the corresponding analyses are designed according to conventionally used parameters similar to other pharmaceutical studies. ${ }^{55}$ A responder analysis is included as secondary analysis to identify possible factors (including biomarkers and symptom-based diagnosis) that could lead to more personalised use of astragalus. Besides, we conducted a focus group interview series to explore the expectations of patients and clinicians (both conventional medicine and $\mathrm{CM}$ ) to refine the study design for better clinical translation. ${ }^{54}$ The major limitation of this trial is the open-label nature. The study subjects could not be masked due to the nature of treatment. Since the clinical outcomes under investigation are objective and the outcome assessor is blinded, placebo effect and outcome measurement bias should be minimised. However, subjective outcomes including quality of life could not be assessed.

\section{Author affiliations}

${ }^{1}$ Department of Medicine, The University of Hong Kong, Hong Kong, China ${ }^{2}$ Department of Family Medicine and Primary Healthcare, Hospital Authority Hong Kong West Cluster, Hong Kong, China

${ }^{3}$ Department of Family Medicine and Primary Healthcare, Hospital Authority Hong Kong East Cluster, Hong Kong, China

${ }^{4}$ Department of Medicine, Queen Elizabeth Hospital, Hong Kong, China ${ }^{5}$ School of Chinese Medicine, The University of Hong Kong, Hong Kong, China ${ }^{6}$ Virginia University of Integrative Medicine, Fairfax, Virginia, USA

Acknowledgements We thank Ka Yan Yu and Feona Leung for the clinical service; Pearl Yan, Kam Yan Yu, Dr Johnny Lam, Louise Pun, Sally Lam, Ying Wong and Tommy Lee for the patient coordination and clerical support; Spencer Ng from Information and Technology for the generation and concealment of randomisation sequence; Janice Wong and C\&H Medical Laboratories Ltd. for the independent analysis of clinical samples and all clinical and scientific staffs involved in the patient care and sample analysis. This project is a part of SCHEMATIC Initiative and we thank all the team members of SCHEMATIC for their contribution. We also thank Professor Vincent Chung, Professor Wendy Wong and Dr Jerry Yeung for monitoring this trial as DSMB panel members.

Contributors KWC and SCWT conceived the study. KWC, ASKK, PNT, SCYC, GCWC, MM-YW, KCBT and WFC recruited the patients and provided clinical consultation. WHY provided expert opinion and support in biochemical analysis. LL, Z-JZ and YZ provided expert opinion on the study design. KWC and SCWT drafted the manuscript. All authors involved in the manuscript revision.

Funding This project is made possible in part through the Health and Medical Research Fund (Ref: 12133341, 14151731). The funding organisation had no role in the design and conduct of the study; collection, management, analysis and interpretation of the data; preparation, review or approval of the manuscript and decision to submit the manuscript for publication. 
Competing interests None declared.

Patient consent for publication Not required.

Provenance and peer review Not commissioned; externally peer reviewed.

Supplemental material This content has been supplied by the author(s). It has not been vetted by BMJ Publishing Group Limited (BMJ) and may not have been peer-reviewed. Any opinions or recommendations discussed are solely those of the author(s) and are not endorsed by BMJ. BMJ disclaims all liability and responsibility arising from any reliance placed on the content. Where the content includes any translated material, BMJ does not warrant the accuracy and reliability of the translations (including but not limited to local regulations, clinical guidelines, terminology, drug names and drug dosages), and is not responsible for any error and/or omissions arising from translation and adaptation or otherwise.

Open access This is an open access article distributed in accordance with the Creative Commons Attribution Non Commercial (CC BY-NC 4.0) license, which permits others to distribute, remix, adapt, build upon this work non-commercially, and license their derivative works on different terms, provided the original work is properly cited, appropriate credit is given, any changes made indicated, and the use is non-commercial. See: http://creativecommons.org/licenses/by-nc/4.0/.

\section{ORCID iDs}

Kam Wa Chan http://orcid.org/0000-0002-3175-1574

Sydney Chi Wai Tang http://orcid.org/0000-0002-6862-1941

\section{REFERENCES}

1 Sinclair A, Saeedi P, Kaundal A, et al. Diabetes and global ageing among 65-99-year-old adults: findings from the International Diabetes Federation Diabetes Atlas, 9th edition. Diabetes Res Clin Pract 2020;162:108078.

2 Cho NH, Shaw JE, Karuranga S, et al. IDF diabetes atlas: global estimates of diabetes prevalence for 2017 and projections for 2045. Diabetes Res Clin Pract 2018;138:271-81.

3 Ogurtsova K, da Rocha Fernandes JD, Huang Y, et al. IDF Diabetes Atlas: global estimates for the prevalence of diabetes for 2015 and 2040. Diabetes Res Clin Pract 2017;128:40-50.

4 Thomas MC, Cooper ME, Zimmet P. Changing epidemiology of type 2 diabetes mellitus and associated chronic kidney disease. Nat Rev Nephrol 2016;12:73-81.

5 Tang SCW, Yiu WH. Innate immunity in diabetic kidney disease. Nat Rev Nephrol 2020;16:206-22.

6 Leung CB, Cheung WL, Li PKT. Renal registry in Hong Kong-the first 20 years. Kidney Int Suppl 2015;5:33-8.

7 Jiao F, Wong CKH, Tang SCW, et al. Annual direct medical costs associated with diabetes-related complications in the event year and in subsequent years in Hong Kong. Diabet Med 2017;34:1276-83.

8 Afkarian M, Sachs MC, Kestenbaum B, et al. Kidney disease and increased mortality risk in type 2 diabetes. J Am Soc Nephrol 2013;24:302-8

9 Wen CP, Chang CH, Tsai MK, et al. Diabetes with early kidney involvement may shorten life expectancy by 16 years. Kidney Int 2017;92:388-96.

10 Goh S-Y, Cooper ME. Clinical review: the role of advanced glycation end products in progression and complications of diabetes. J Clin Endocrinol Metab 2008;93:1143-52.

11 Forbes JMet al. Role of advanced glycation end products in diabetic nephropathy. J Am Soc Nephrol 2003;14:254S-8.

12 Pichler R, Afkarian M, Dieter BP, et al. Immunity and inflammation in diabetic kidney disease: translating mechanisms to biomarkers and treatment targets. Am J Physiol Renal Physiol 2017;312:F716-31.

13 Navarro-González JF, Mora-Fernández C. The role of inflammatory cytokines in diabetic nephropathy. J Am Soc Nephrol 2008;19:433-42.

14 Navarro-González JF, Mora-Fernández C, Muros de Fuentes M, et al. Inflammatory molecules and pathways in the pathogenesis of diabetic nephropathy. Nat Rev Nephrol 2011;7:327-40.

15 Alicic RZ, Rooney MT, Tuttle KR. Diabetic kidney disease: challenges, progress, and possibilities. Clin J Am Soc Nephrol 2017;12:2032-45.

16 Premaratne E, Verma S, Ekinci El, et al. The impact of hyperfiltration on the diabetic kidney. Diabetes Metab 2015;41:5-17.

17 Tonneijck L, Muskiet MHA, Smits MM, et al. Glomerular hyperfiltration in diabetes: mechanisms, clinical significance, and treatment. J Am Soc Nephrol 2017;28:1023-39.

18 Palatini P. Glomerular hyperfiltration: a marker of early renal damage in pre-diabetes and pre-hypertension. Nephrol Dial Transplant 2012;27:1708-14.
19 Yiu WH, Li RX, Wong DWL, et al. Complement C5a inhibition moderates lipid metabolism and reduces tubulointerstitial fibrosis in diabetic nephropathy. Nephrol Dial Transplant 2018;33:1323-32.

20 Alsaad KO, Herzenberg AM. Distinguishing diabetic nephropathy from other causes of glomerulosclerosis: an update. J Clin Pathol 2007;60:18-26.

21 Hsu T-W, Liu J-S, Hung S-C, et al. Renoprotective effect of reninangiotensin-aldosterone system blockade in patients with predialysis advanced chronic kidney disease, hypertension, and anemia. JAMA Intern Med 2014:174:347-54.

22 Nistor I, De Sutter J, Drechsler C, et al. Effect of renin-angiotensinaldosterone system blockade in adults with diabetes mellitus and advanced chronic kidney disease not on dialysis: a systematic review and meta-analysis. Nephrol Dial Transplant 2018;33:12-22.

23 Palmer SC, Mavridis D, Navarese E, et al. Comparative efficacy and safety of blood pressure-lowering agents in adults with diabetes and kidney disease: a network meta-analysis. Lancet 2015;385:2047-56.

24 Chan GCW, Tang SCW. Proteinuria reaffirmed as a risk modifier in diabetic chronic kidney disease. Nephrol Dial Transplant 2018;33:1873-4.

25 Chan GCW, Tang SCW. Diabetic nephropathy: landmark clinical trials and tribulations. Nephrol Dial Transplant 2016;31:359-68.

26 Lin M-Y, Chiu Y-W, Chang J-S, et al. Association of prescribed Chinese herbal medicine use with risk of end-stage renal disease in patients with chronic kidney disease. Kidney Int 2015;88:226

27 Hsieh CF, Huang SL, Chen CL, et al. Non-aristolochic acid prescribed Chinese herbal medicines and the risk of mortality in patients with chronic kidney disease: results from a population-based follow-up study. BMJ Open 2014;4:e004033.

28 Li Q, Zhang H-M, Fei Y-T. [Treatment of diabetic nephropathy by integrative medicine: a multi-center prospective cohort study]. Zhongguo Zhong Xi Yi Jie He Za Zhi 2012;32:317-21.

29 Zhang HW, Lin ZX, Xu C, et al. Astragalus (a traditional Chinese medicine) for treating chronic kidney disease. Cochrane Database Syst Rev 2014;22:CD008369.

30 Li M, Wang W, Xue J, et al. Meta-analysis of the clinical value of Astragalus membranaceus in diabetic nephropathy. $J$ Ethnopharmacol 2011;133:412-9.

31 Yi-hong LIU, Hao-ming T. Effect of astragaulus membranaceus on diabetic nephropathy: a systematic review. Chinese J EvidenceBased Med 2007;7:715-27.

32 Gui D, Guo Y, Wang F, et al. Astragaloside IV, a novel antioxidant, prevents glucose-induced podocyte apoptosis in vitro and in vivo. PLoS One 2012;7:22.

33 Sun L, Li W, Li W, et al. Astragaloside IV prevents damage to human mesangial cells through the inhibition of the NADPH oxidase/ROS/ Akt/NF- $\kappa B$ pathway under high glucose conditions. Int $\mathrm{J} \mathrm{Mol} \mathrm{Med}$ 2014;34:167-76.

34 Chan KW, Kwong ASK, Chan GCW, et al. Semi-individualised Chinese Medicine Treatment for Diabetic Kidney Disease - From users' perspectives to SCHEMATIC trial interim result and potential mechanisms. Adv Integr Med 2019;6:S12.

35 Chan KW, Ip TP, Kwong ASK, et al. Semi-individualised Chinese medicine treatment as an adjuvant management for diabetic nephropathy: a pilot add-on, randomised, controlled, multicentre, open-label pragmatic clinical trial. BMJ Open 2016;6:e010741.

36 WHO. General guidelines for methodologies on research and evaluation of traditional medicine. Geneva: World Health Organisation, 2000.

37 Levey AS, Bosch JP, Lewis JB, et al. A more accurate method to estimate glomerular filtration rate from serum creatinine: a new prediction equation. modification of diet in renal disease study group. Ann Intern Med 1999;130:461-70.

38 Glassock RJ, Warnock DG, Delanaye P. The global burden of chronic kidney disease: estimates, variability and pitfalls. Nat Rev Nephrol 2017;13:104-14.

$39 \operatorname{Sim} \mathrm{J}$, Lewis M. The size of a pilot study for a clinical trial should be calculated in relation to considerations of precision and efficiency. $J$ Clin Epidemiol 2012;65:301-8.

40 Moore CG, Carter RE, Nietert PJ, et al. Recommendations for planning pilot studies in clinical and translational research. Clin Transl Sci 2011;4:332-7.

41 Pavlou M, Ambler G, Seaman SR, et al. How to develop a more accurate risk prediction model when there are few events. BMJ 2015;351:h3868.

$42 \mathrm{BC}$ L. The combined treatment of Astragalus and Vasartan for early stage diabetic kidney disease. China Foreign Med Treat 2011;23:98-100.

43 Huang H, Gao J. Astragalus pharmacological properties and use of research in diabetic nephropathy. J Pract Tradit Chinese Inter Med 2011;25:80-2. 
44 Ford I, Norrie J. Pragmatic trials. N Engl J Med 2016;375:454-63.

45 Grams ME, Sang Y, Ballew SH, et al. Evaluating glomerular filtration rate slope as a surrogate end point for ESKD in clinical trials: an individual participant meta-analysis of observational data. J Am Soc Nephrol 2019;30:1746-55.

46 Cheng C-W, Wu T-X, Shang H-C, et al. CONSORT extension for Chinese herbal medicine formulas 2017: recommendations, explanation, and elaboration. Ann Intern Med 2017;167:112-21.

47 Krolewski AS, Skupien J, Rossing P, et al. Fast renal decline to end-stage renal disease: an unrecognized feature of nephropathy in diabetes. Kidney Int 2017;91:1300-11.

48 Looker HC, Colombo M, Hess S, et al. Biomarkers of rapid chronic kidney disease progression in type 2 diabetes. Kidney Int 2015;88:888-96.

49 Zheng X. Guiding principle of clinical research on new drugs of Chinese medicine (trial). Beijing: China Medical Science Press, 2002.

50 Umanath K, Lewis JB. Update on diabetic nephropathy: core curriculum 2018. Am J Kidney Dis 2018;71:884-95.
51 Chan KW. Integrative management for diabetic kidney disease patients' and clinicians' perspectives, clinical effectiveness and possible mechanisms (PhD thesis). Hong Kong: The University of Hong Kong, 2018.

$52 \mathrm{Ma} \mathrm{Y-C,} \mathrm{Zuo} \mathrm{L,} \mathrm{Chen} \mathrm{J-H,} \mathrm{et} \mathrm{al.} \mathrm{Modified} \mathrm{glomerular} \mathrm{filtration} \mathrm{rate}$ estimating equation for Chinese patients with chronic kidney disease. J Am Soc Nephrol 2006;17:2937-44.

53 Levey AS, Stevens LA, Schmid CH, et al. A new equation to estimate glomerular filtration rate. Ann Intern Med 2009;150:604-12.

54 Chan KW, Lee PW, Leung CPS, et al. Patients' and clinicians' expectations on integrative medicine services for diabetes: a focus group study. BMC Complement Med Ther 2020;20:205.

55 Heerspink HJL, Parving H-H, Andress DL, et al. Atrasentan and renal events in patients with type 2 diabetes and chronic kidney disease (SONAR): a double-blind, randomised, placebo-controlled trial. Lancet 2019;393:1937-47. 